\title{
Gradhiva
}

GRADHIV

Revue d'anthropologie et d'histoire des arts

\section{Avec Alain Epelboin et Jean-Charles Coulon. D'hier à aujourd'hui : enquêtes sur les talismans et les manuscrits de sciences occultes en Islam}

Alain Epelboin, Jean-Charles Coulon et Andrea-Luz Gutierrez-Choquevilca

\section{(2) OpenEdition}

Journals

Édition électronique

URL : https://journals.openedition.org/gradhiva/5638

DOI : $10.4000 /$ gradhiva. 5638

ISSN : 1760-849X

Éditeur

Musée du quai Branly Jacques Chirac

Édition imprimée

Date de publication : 24 mars 2021

Pagination : 159-173

ISBN : 978-2-35744-132-3

ISSN : 0764-8928

Référence électronique

Alain Epelboin, Jean-Charles Coulon et Andrea-Luz Gutierrez-Choquevilca, « Avec Alain Epelboin et Jean-Charles Coulon. D'hier à aujourd'hui : enquêtes sur les talismans et les manuscrits de sciences occultes en Islam », Gradhiva [En ligne], 32 | 2021, mis en ligne le 02 avril 2021, consulté le 25 mai 2021. URL : http://journals.openedition.org/gradhiva/5638 ; DOI : https://doi.org/10.4000/gradhiva 5638

(c) musée du quai Branly 


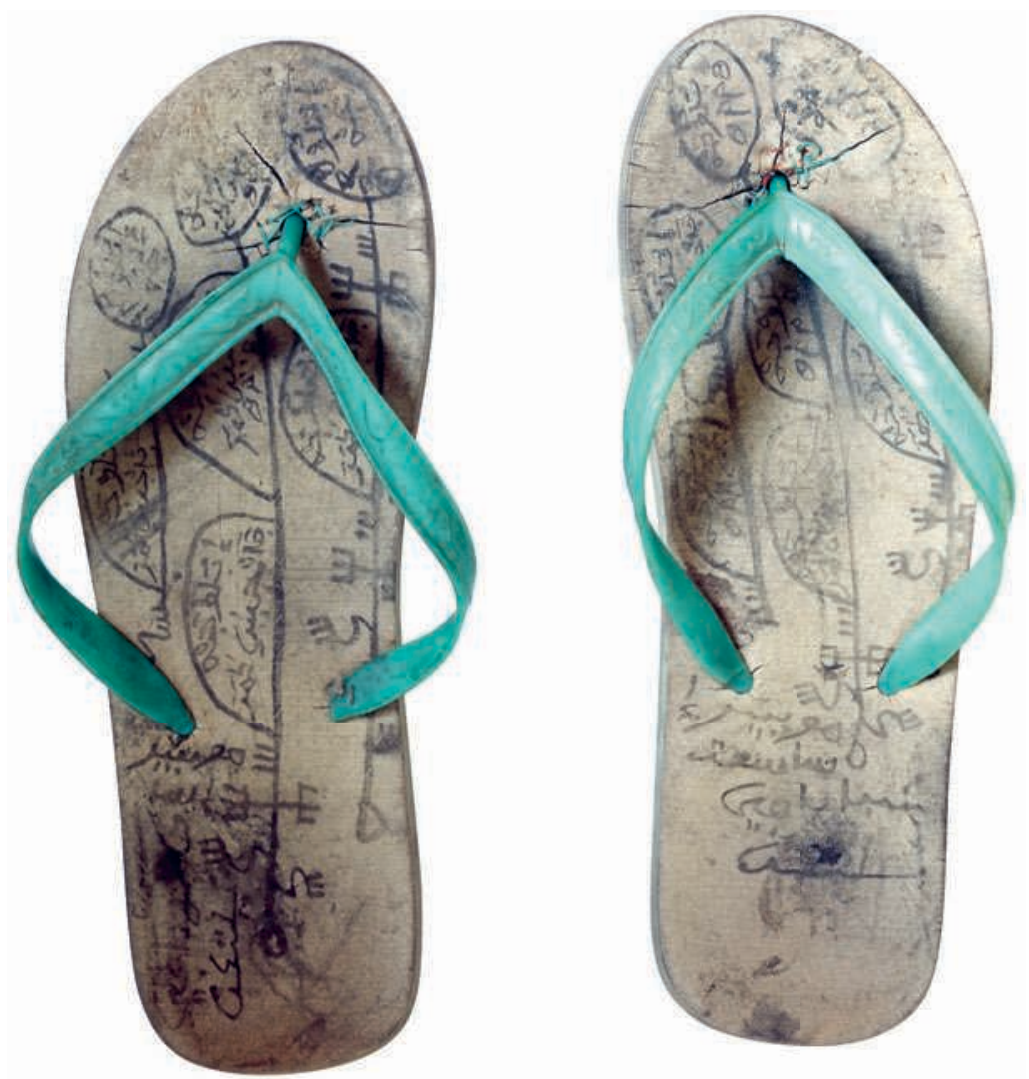


Avec Alain Epelboin et Jean-Charles Coulon.

D'hier à aujourd'hui: enquêtes sur

les talismans

et les manuscrits

de sciences occultes en Islam

$$
\begin{gathered}
\text { Andrea-Luz } \\
\text { Gutierrez-Choquevilca }
\end{gathered}
$$


Contrairement à la théologie ou la philosophie, les sciences occultes ont souvent été abordées avec dédain et mépris dans la tradition savante orientaliste au XIXé Điècle et au début du $x x^{e}$. Pourtant, ces sciences étaient très importantes, notamment parmi les élites, comme en témoigne le mécénat exercé par des califes et des souverains dès le ville [iiècle. Ce succès a persisté aux époques mameloukes et ottomanes, qui nous ont légué un grand nombre de manuscrits. L'apparition de la lithographie a cependant permis une plus large di पusion de ces savoirs, et, par exemple, le Shams al-ma 'arif (Le Soleil des connaissances) attribué à al-BDn\$(m. 1225 ou 1232) est devenu un véritable manuel indispensable à tous les praticiens. II s'agit d'un des ouvrages fondamentaux des fabricants de talismans en Afrique de I'Ouest. Une enquête inaugurée par la découverte d'amulettes et d'écrits talismaniques dans une décharge à ordures de Dakar retrace les origines mystérieuses de ces talismans pour mettre au jour leurs hypotextes, issus de manuscrits de sciences occultes médiévaux. Un double regard sur ces objets-textes énigmatiques, celui de l'ethnologue Alain Epelboin et de I'historien Jean-Charles Coulon, révèle les liens qui se tissent entre l'art des écrits talismaniques, l'islam et certains savoirs ésotériques plus anciens.

\begin{abstract}
A.-L. G.-C.: Alain Epelboin, vous êtes médecin anthropologue, chercheur retraité du CNRS au Muséum national d'histoire naturelle. Avant de devenir «savant» dans le système de pensée de vos interlocuteurs, vous avez exercé en Afrique sub-saharienne.

Dans un lieu improbable, Mbeubeuss, la grande décharge à ordures de Dakar, vous avez depuis les années 1980 découvert l'existence d'objets fascinants: des milliers d'amulettes et de talismans abandonnés par leurs usagers, dans un excellent état de conservation. Certains de ces objets sont réunis dans la Collection AIEp (1983-2018), sise au musée de l'Homme, qui a donné lieu à de nombreuses expositions et qui est consultable virtuellement 1 . Pourriez-vous nous dire quelques mots de votre rencontre avec ces objets énigmatiques?
\end{abstract}

A. E.: Ma découverte de Mbeubeuss, la grande décharge à ordures de Dakar, date des années 1980, lors d'un proǵramme de recherche «urbanisation-santé» de l'Office de la recherche scientifique et technique outre-mer (Orstom). J'étais déjà très spécialisé dans l'anthropologie des déchets corporels et ménagers, inaugurée par les travaux de Marcel Mauss, Mary Douǵlas et Roger-Henri Guerrand et prolongée par la mise en place d'une méthodologie complémentaire de celles employées par les ethnologues, l'observation des traces, des déchets, c'est-à-dire «une archéologie du temps présent» : elle permet de définir les normes autochtones de dyades et polydyades fondamentales, constituées par les notions de haut/bas, droite/gauche, masculin/féminin, sec/humide, aval/amont, propre/sale, pureté/souillure, etc.

Par ailleurs, probablement en raison de mon histoire personnelle et familiale, marquée par les camps de concentration nazis et la crainte d'une apocalypse nucléaire, j'ai été captivé par cette population de travailleurs de l'ordure, un microcosme de quelques centaines de résidents, vivant de la décharge et dans la décharge: migrants ruraux saisonniers, chômeurs urbains devenus «collecteurs auto-entrepreneurs», individus déclassés en raison de malheurs à répétition, d'addictions, de mœurs sexuelles différentes, de pathologies mentales légères ou lourdes, parfois des délinquants cherchant à se faire oublier...
Dans cet environnement «postapocalyptique», les normes hiérarchiques coutumières, ethnie, caste, religion, etc., sont remises en question. On respecte les aînés et les vieux/vieilles s'ils sont dignes de respect, on cultive la dignité de soi et aussi les solidarités humanistes inter pares, ce qui n'exclut pas certaines violences. Pour résumer, je dirais que contrairement à l'idée préconçue qui amène à considérer les récupérateurs d'ordures comme des parias, des «intouchables» méprisables, j'ai découvert une société marǵinale, donneuse de leçons d'humanité, peut-être aussi un laboratoire social de futurs possibles, même en cas d'apocalypse meurtrière et destructrice. Et la stimulation par mes soins de la collecte des seules «matières-déchets » qui n'étaient pas récupérées, ou seulement de manière anedoctique, à savoir les amulettes et les objets artisanaux traditionnels et magiques, m'a permis, selon les mots de ma collègue Bernadette Lizet, de transformer une névrose obsessionnelle de collectionneur d'objets depuis l'adolescence en objet, voire en découverte scientifique.

A.-L. G.-C.: Cette découverte scientifique et humaine force l'admiration. Au-delà de cette rencontre, c'est aussi le regard 
croisé de l'ethnologue et de I'historien qui est à l'origine de la redécouverte de ces objets. Votre collaboration avec I'islamologue Constant Hamès et I'historien médiéviste JeanCharles Coulon, spécialiste de la littérature magique en Islam, révèle la fécondité d'une enquête portant sur la mise au jour des écrits talismaniques islamiques extraits des amulettes de la décharge de Mbeubeuss.

A. E. : Nous avons extrait des milliers de ceintures en cuir ou de pochons rectangulaires ou carrés, un énorme corpus de manuscrits sur papier: l'une des caractéristiques de ces calliǵraphies, dans des sociétés essentiellement perçues comme relevant de traditions orales, c'est leur reproduction, pour une grande part à l'identique, depuis les débuts de l'islam et pour certaines depuis les temps anté-islamiques. Cela n'exclut pas bien sûr le recours à l'inventivité, selon la personnalité du prescripteur et l'adaptation aux besoins des différentes époques et sociétés. De ce fait, les allers-retours entre les disciplines, ici entre l'historien et l'ethnologue/ethnomédecin sont indispensables et fructueux...

\section{A.-L. G.-C.: Pourriez-vous nous présenter I'histoire de la collection d'AlEp? De quoi sont constituées les pièces de cette collection?}

A. E.: On peut classer ǵrossièrement ces artefacts maǵiques en trois catégories $\mathbf{2}$ :

- des objets d'obédience africaine stricte, avec des traits anté-islamiques ou chrétiens, ethniques et/ou transethniques, basés sur l'emploi cumulatif ou analogique de matières minérales, marines, animales (corne, cuir, os, poils, plumes...), végétales (bois, écorces, racines, feuilles en morceaux ou en poudre), industrielles (métaux, miroirs, bouteilles, fils, tissus...), objets miniatures. Par exemple, l'emploi fréquent de cuir de varan vise à s'approprier les vertus ethnoéthologiques de l'animal, qui semble très lent et facile à attraper, alors qu'il s'enfuit dès qu'on tente de le prendre: c'est cette vertu qu'on va utiliser, associée à des versets sur la dissimulation, la réussite, la victoire, la neutralisation des ennemis.

- des objets islamo-africains, c'est-àdire combinant les artefacts des traditions africaines précités et des écritures de fraǵments coraniques inscrits sur des papiers pliés dans lesquels peuvent être insérées des matières végétales, animales, minérales, qui par analogie et accumulation renforceront les pouvoirs qui ont été mis en branle par l'écriture. Le manuscrit, remis plié au client, bobiné de fils de couleurs spécifiques ou enveloppé dans un tissu d'une couleur déterminée, est éventuellement confié à un cordonnier avec des indications de fabrication, par exemple quant au nombre de boucles des ceintures ou de cauris apparents, correspondant dans la plupart des cas à des chiffres efficients valorisés dans les écrits. L'écriture peut être réalisée sur des tissus, parfois des cuirs, des métaux, des bois... Ces mêmes assemblages de lettres, de chiffres, de mots, de phrases, de symboles peuvent aussi être récités sur de l'eau, un corps animal ou humain, lors de la confection d'un nœud d'arrêt, lors de la fermeture d'un cadenas... Parfois l'écrit, y compris des carrés maǵiques et des figures complexes sur une tablette coranique ou sur un papier, est dissout dans de l'eau et employé en onction ou en remède ingéré, ou encore dispersé dans l'espace.

- des objets, d'obédience islamique stricte, constitués exclusivement des calligraphies précitées, caractérisées par le refus de mêler l'islam à des croyances africaines considérées comme païennes.

\section{A.-L. G.-C.: Ces talismans sont} des objets hybrides composés à partir d'une grande variété de supports et de matières. Une logique de l'accumulation semble à l'œuvre dans leur composition, qui fait écho à la structure de répétition que I'on retrouve dans le contenu même des écrits talismaniques. Dans I'un des talismans, celui de la laveuse par exemple, les images du scorpion et du serpent sont juxtaposées à des carrés magiques, sur lesquels sont inscrits des chiffres et des noms. La fabrique de ces objets rituels révèle des réseaux de correspondances subtils entre l'image, la parole et l'inscription Iorsqu'elle existe. Quelle relation I'usager entretient-il avec le contenu de ces amulettes? L'usager est-il aussi lecteur de ces talismans?

A. E.: Les utilisateurs de ces manuscrits talismaniques, dans la grande majorité des cas, sont illettrés et ne sont pas capables de décrypter ce qui est écrit en dehors des noms d'Allah, de Mahomet, de quelques éléments d'écriture simples. Et la plupart du temps, le prescripteur remet l'objet fini à son client sans que celui-ci puisse prendre connaissance de son contenu, car celui-ci est «non visible», plié, bobiné de fil et cousu dans une gaine de tissu. Le secret, quant au contenu de l'amulette et aux paroles prononcées ou écrites, est de rèǵle. Mais on ne peut pas totalement dissocier l'écriture de l'oralité puisque les mêmes écrits peuvent être prononcés. Avec le temps, l'indication princeps de l'amulette, c'est-à-dire l'intentionnalité du scribe qui a composé son manuscrit, peut s'estomper, remplacée par les croyances en des pouvoirs spécifiques que son usager lui a attribués en fonction des épreuves surmontées: l'usage et le vécu de l'amulette vont transformer les indications de celle-ci et les vertus qui lui sont attribuées. L'efficacité de ces objets, au-delà de la croyance aux vertus intrinsèques de ses composants, tient à leur caractère mémoriel: tels les objets transitionnels si efficaces chez les tout-petits humains, ils représentent des mémoires conscientes et inconscientes d'événements, de lieux, de temps, de personnes, d'émotions ${ }^{3}$.

\section{A.-L. G.-C.: Les indications de ces amulettes sont variables et évolutives. À quel objet pensez-vous?}

A. E. : L'objet qui me plaît le plus, c'est celui où il n'y a pas d'écriture visible mais sur lequel il y a peut-être eu des écritures qui ont été prononcées, à un moment donné. C'est un flacon industriel d'Ajax ammoniaqué, exposé dans le parcours permanent du musée de l'Homme, en plastique blanc avec un bouchon vissant rouge, transformé en contenant maǵique par son fabricant par l'ajout de tissus rouge, blanc, noir, de cauris et de cornes. Il a contenu un liquide lustral, probablement employé dans un combat de lutte sénégalaise. On peut supposer que des formules talismaniques ont été prononcées. Lors du réemploi de ce flacon de détergent industriel blanc à odeur d'ammoniac, son usage princeps était vraisemblablement iǵnoré : et plus encore, la campagne publicitaire qui avait assuré son succès, puisqu'il s'agissait d'une tornade blanche qui sortait du flacon et qui faisait le ménaǵe à la place de la ménagère, en écho au mythe de la lampe d'Aladdin et du djinn qui en sort lorsqu'on la frotte. Il s'agissait done d'un objet industriel, dont la promotion auprès de publics européens faisait référence à un mythe moyen-oriental pour construire son efficacité. Celle-ci reposait également sur la popularité du pouvoir «hygiénique» attribué à l'ammoniac, ainsi que sur son emploi sous forme de sels d'ammoniac, 


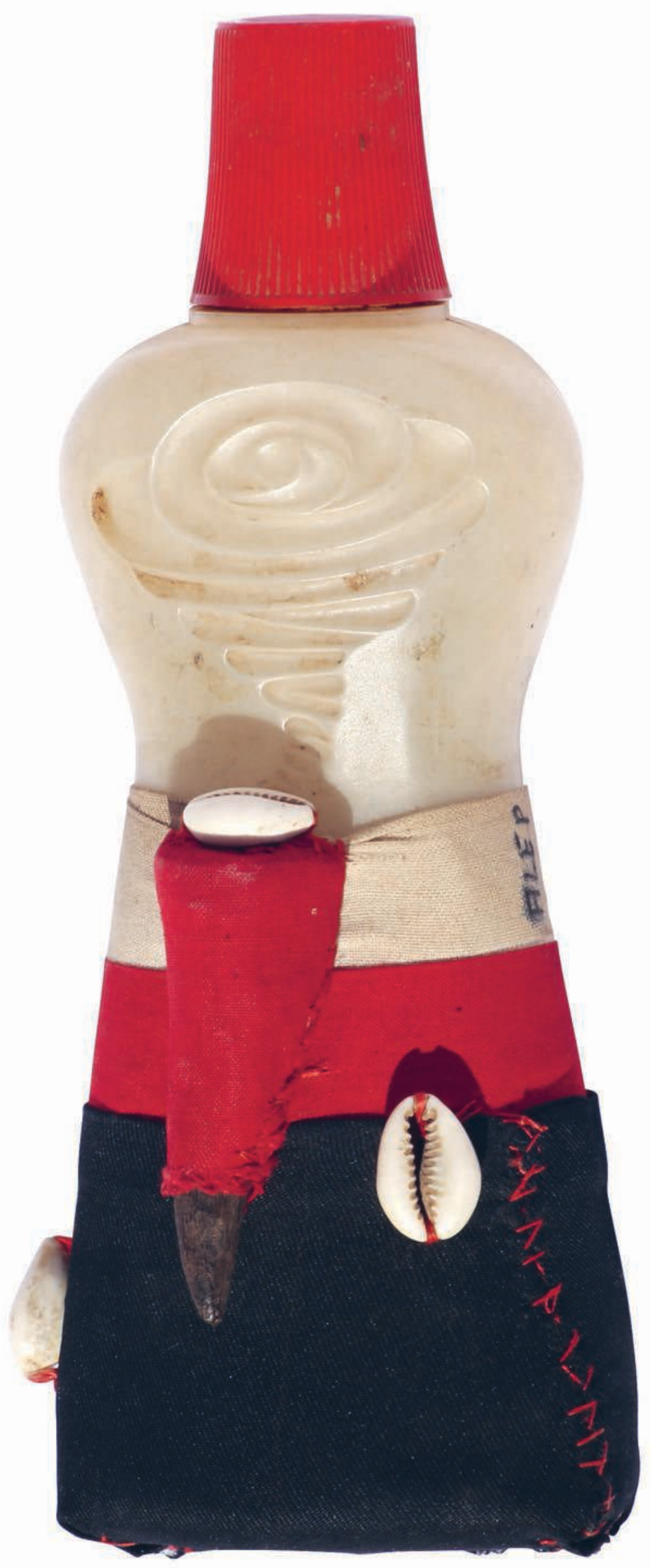

Bouteille d'Ajax. Collection ALEPघamulettes et objets magiques collectés dans les ordures (Mbebess Joal, Mbour, Sénégal, Afrique, Europe) par Alain Epelboin, 1983-2014. ALEP00536. 


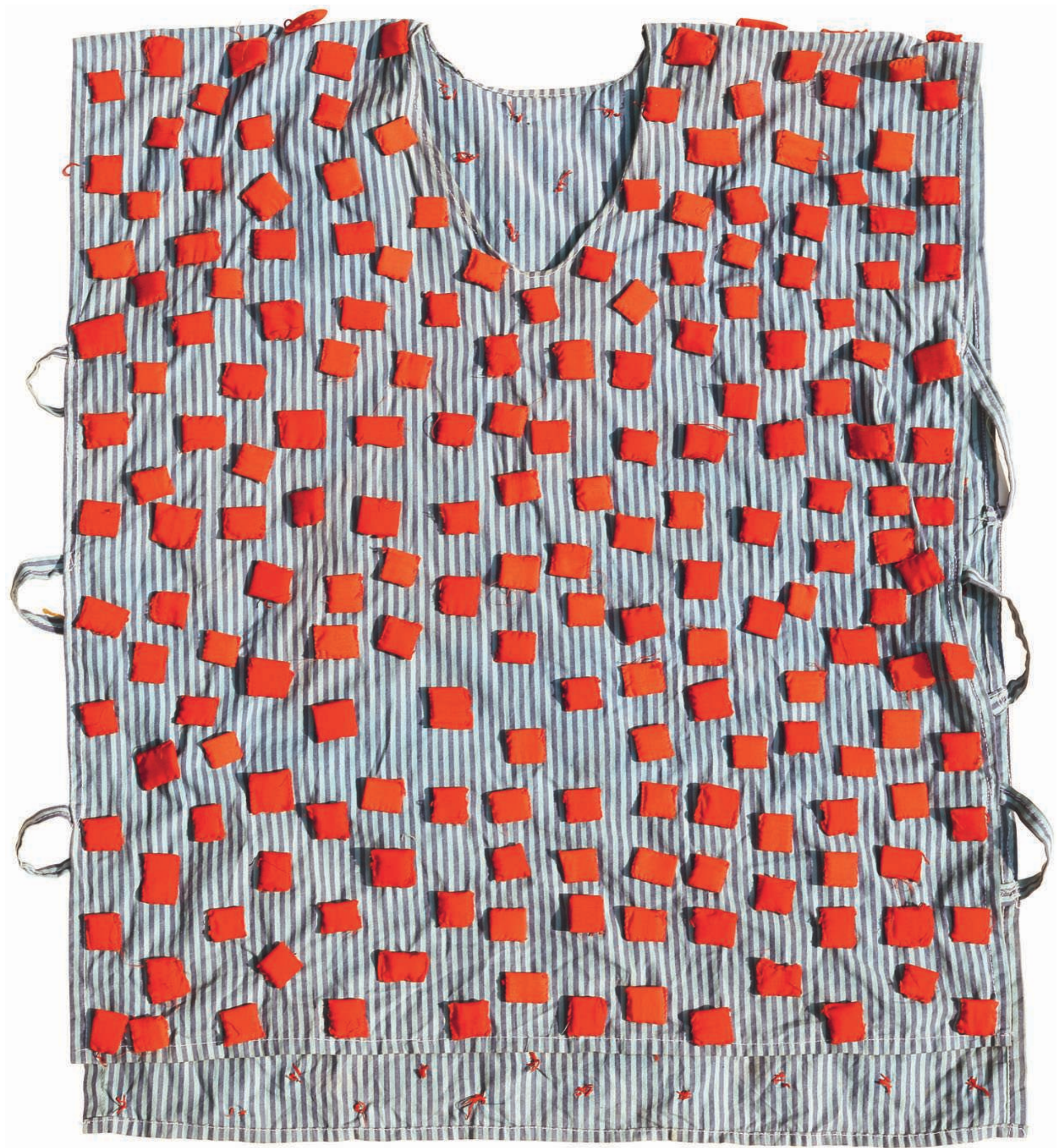

Tunique talismanique en cotonnade industrielle bleue parsemée de très nombreux écrits pliés en carré, gainés dans un tissu rouge, 60 ' $71 \mathrm{~cm}$.

Collection ALEPEamulettes et objets magiques collectés dans les ordures (Mbebess Joal, Mbour, Sénégal, Afrique, Europe) par Alain Epelboin, 1983-2014. ALEP40001. 
que l'on faisait respirer alors à une femme évanouie, thérapie d'un syndrome culturel genré. Et donc, cet objet «modeste» se retrouve à la croisée de signifiés et de signifiants divers: un flacon étanche, un produit hygiénique, un remède "genré » et des djinns moyen-orientaux ou sénégalais asservis par des humains à leurs fins personnelles.

\section{A.-L. G.-C.: Les formules} talismaniques viennent activer le pouvoir de substances, autrement sans effet sur leur destinataire. L'efficacité d'un talisman ne semble pas dépendre du caractère visible de ses inscriptions. C'est intéressant. Mais malgré tout, même dans le cas de ce flacon d'Ajax que vous évoquez, c'est la performativité des paroles énoncées sur l'objet qui est au fondement de son étrange pouvoir. La contiguïté entre l'objet et la parole magique est déterminante. Cela évoque la forme d'un palimpseste. Chaque amulette se réfère à un hypotexte constitué par des formules magiques énoncées oralement, dissimulées sur un manuscrit ou inscrites à la surface de l'objet. Qui sélectionne le texte source et selon quels critères?

A. E.: Ce sont les prescripteurs, les tradipraticiens d'obédience islamique, dont il faut distinguer les niveaux de savoir, depuis la personne à peine lettrée, qui est copiste de manuscrits, et à présent des photocopies qu'il possède, obtenus par exemple auprès de maîtres ou d'amis lors d'itinéraires diagnostiques et thérapeutiques personnels. Il va les recopier minutieusement, y compris les fautes originelles qu'il n'a pas été capable de distinguer, et en y ajoutant ses propres malfaçons. À l'autre bout de la hiérarchie, il y a le lettré très savant, qui connaît le Coran par cœur, auquel on attribue des pouvoirs de voyance, parfois même sans aucun support matériel de divination.

A.-L. G.-C.: Je propose de faire un saut dans le temps pour examiner les textes sources de quelques-uns de ces talismans retrouvés à Mbeubeuss. Jean-Charles Coulon, en tant qu'historien médiéviste et arabisant, vous avez consacré vos recherches à l'étude de la genèse de l'un des plus grands manuels de magie islamique, le Shams al-ma 'ārif (Le Soleil des connaissances), un traité de sciences occultes attribué au maître soufi al-Būnī. II s'agit en réalité d'un texte aux origines mal connues, une immense compilation, comportant des références au Coran, à la tradition prophétique et aux traditions magiques, mystiques et kabbalistiques juives, chrétiennes et musulmanes. À la différence d'Alain Epelboin, vous vous intéressez davantage à la généalogie de ces écrits talismaniques qu'à leurs usages contemporains. Que sait-on de la composition et de la transmission de ces textes au Moyen Âge?

J.-C. C.: Ce qui m'a beaucoup intéressé dans ce travail, c'est de comprendre que le rapport au texte change selon les époques. Je me consacre en effet à la période de composition d'écrits devenus des textes de référence, comme le Shams al-ma'āif (Le Soleil des connaissances), attribué à al-Būnī (m. 1225 ou 1232) ${ }^{4}$. Quand j'ai commencé mes recherches de doctorat, j'étais parti du constat qu'il s'agissait d'un texte lithographié au XIX siècle, très courant dans le monde arabe de nos jours, et quasiment tous les travaux que je pouvais lire à ce sujet étaient ceux d'anthropologues, ethnologues, ethnographes, mais non d'historiens ${ }^{5}$. On parle d'un texte très connu, extrêmement diffusé, que les populations - qualifiées d' «indigèènes » dans le vocabulaire colonial - se sont approprié. Les ethnologues de l'époque affirmaient ainsi qu'il s'aǵissait d'un savoir populaire, bien loin d'une élite savante. En réalité, mes recherches m'ont conduit à penser que le texte avait plutôt été composé dans des milieux proches du pouvoir, avec beaucoup de références à une culture de l'élite, par exemple à la littérature arabe, mais fonctionnant par clins d'œil : sans connaissance à la fois de la littérature arabe, de la philosophie et de l'exégèse coranique, on passe à côté de nombreuses allusions et références. Or, dans la transmission de ce texte, cette connaissance préalable nécessaire a proǵressivement cédé le pas à une visée bien plus pratique. Ainsi, la version lonǵue de ce texte, probablement composée au $\mathrm{XVI}^{\mathrm{e}}$ siècle, qui fut lithographiée au XIX ${ }^{\mathrm{e}}$ siècle et continue d'être rééditée régulièrement, accompaǵne beaucoup plus le lecteur dans la pratique, selon moi, que les versions initiales qui nécessitaient davantage de connaissances préalables pour pouvoir en saisir toutes les dimensions.

Pour prendre un autre exemple, les textes du «talisman de la laveuse» - dont des exemplaires contemporains se trouvent dans la collection AlEp - mettent en scène le calife Hārūn al-Rashīd, victime du sortilège d'une servante: le talisman de cette dernière est découvert dans ses cheveux après sa mort par la servante chargée de laver son corps ${ }^{6}$. Il y a dans les textes des mentions d'autres concubines historiquement documentées de Hārūn al-Rashīd, ce qui vise à mieux intégrer le talisman dans l'histoire de ce calife. Ce calife apparaît en outre dans Les Mille et Une Nuits, tout comme dans d'autres textes de littérature? ${ }^{7}$. On a ainsi beaucoup de passaǵes dans le Shams al-ma'ōrif où les exemples s'appuient sur des événements ou des personnages historiques sans doute inconnus de ceux qui ne disposaient pas d'une solide formation lettrée ${ }^{8}$. Ce texte, qui est une compilation de sciences occultes, a eu un tel succès dans les cours mameloukes et ottomanes qu'on en a gardé de nombreux et très riches manuscrits. Mais il a accédé à un lectorat bien plus large avec le passage à la lithoǵraphie: à partir de ce moment-là, l'engouement a atteint tous ceux qui pouvaient s'acheter un livre, ce qui était impossible pour les manuscrits, à une époque où, évidemment, la copie est chère et rare. Toutefois, on manque d'informations concernant l'éventualité que des utilisateurs qui n'appartenaient pas à cette culture lettrée aient pu avoir recours à ce texte durant le Moyen Âge. Y a-t-il eu une plus grande diffusion? Nous n'avons pas de réelles indications, et nous ne savons pas qui pouvait accéder à ce genre de manuscrits ou ce genre de savoirs qui - normalement - présupposaient une initiation en dehors de ces élites.

A.-L. G.-C.: La connaissance des manuscrits de sciences occultes arabes est originellement réservée à une élite savante et lettrée au Moyen Âge. Ce n'est qu'à une période plus récente que ces textes ont fait l'objet d'une diffusion plus large, au point d'infiltrer toutes les sphères de la société africaine contemporaine. Quelles sources permettent à I'historien d'avoir accès aux usages sociaux et aux conditions de fabrication des talismans à écritures? 


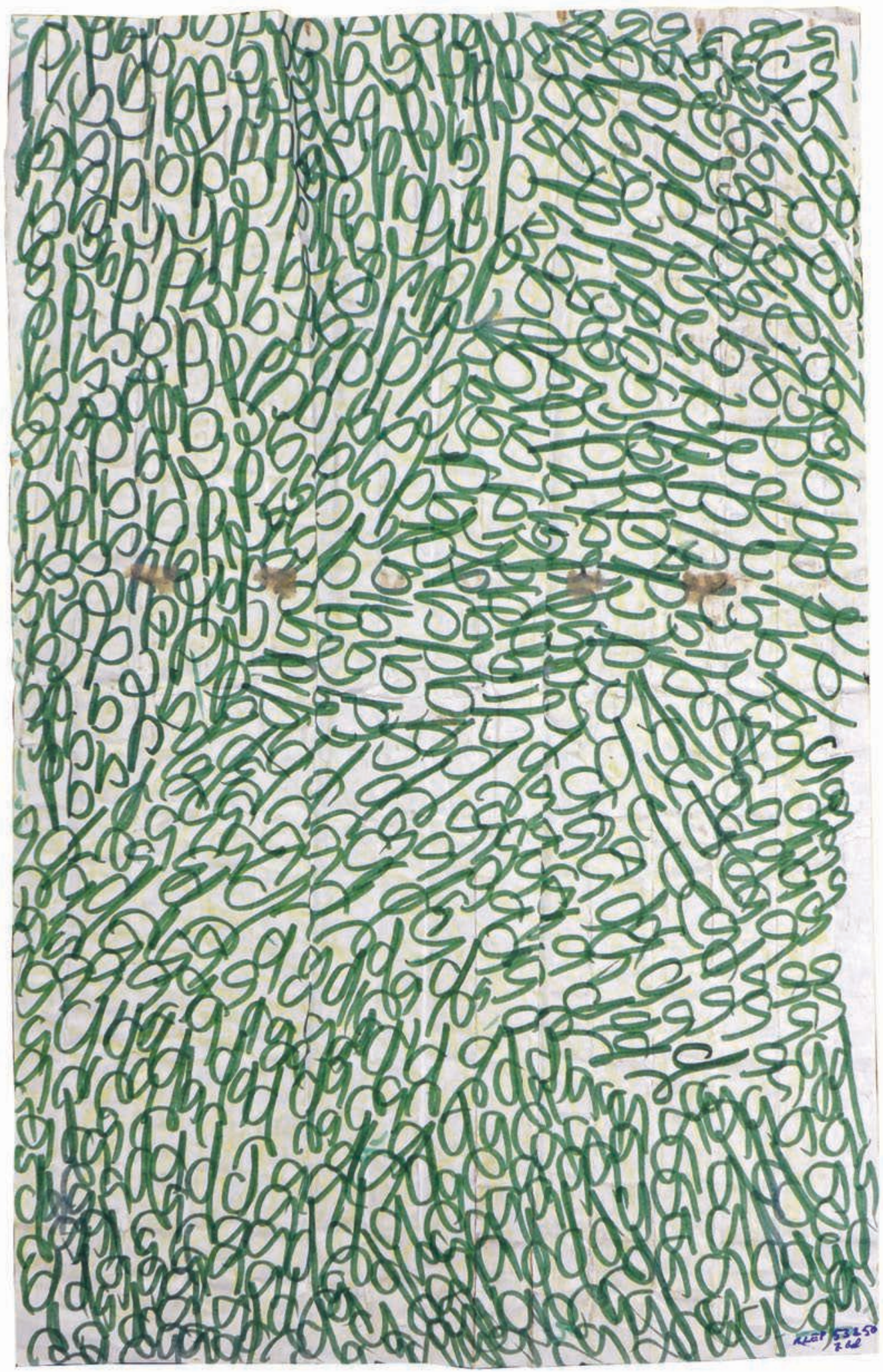

Collection ALEPEamulettes et objets magiques collectés dans les ordures (Mbebess Joal, Mbour, Sénégal, Afrique, Europe) par Alain Epelboin, 1983-2014. ALEP53250. 
J.-C. C.: Il y a des objets tels que des talismans écrits sur papyrus, sur parchemin, sur papier, il y a des tuniques talismaniques. Par exemple, des grands vizirs ou généraux ottomans ou certains souverains avaient des tuniques avec beaucoup d'écritures talismaniques dont certaines sont exposées dans des musées, comme à Istanbul 9 . Des objets comme des bols ou des miroirs talismaniques sont également conservés ${ }^{10}$. Ensuite, sauf dans le cas d'objets vraiment fastueux, telles les tuniques des grands généraux, pour lesquels nous savons qu'ils étaient accessibles à des individus hauts placés dans l'échelle sociale, nous sommes assez mal renseiǵnés sur les personnes qui fabriquaient ces objets et leurs destinataires. Une autre difficulté qui se pose aussi à l'historien est l'identification précise des textes sur lesquels se sont fondés les artisans. Est-ce, par exemple, le Shams al-ma'ärif ou est-ce l'un de ces nombreux autres textes qui circulaient et qui sont encore très mal connus, parce qu'ils n'ont toujours pas été édités et n'existent qu'à l'état de manuscrits? C'est une difficulté de ce travail-là. On a en tout cas des objets qui attestent que ces savoirs étaient mis en pratique via des amulettes et des talismans.

D'autres sources que l'historien peut exploiter au sujet des pratiques sont les textes juridiques, à l'instar des recueils de fatwas, réponses données à des problèmes juridiques, où sont souvent posées aux juges ou aux jurisconsultes des questions sur l'usaǵe de ce génre d'objet. Par exemple: est-il autorisé d'utiliser un objet pour se protéger ou pour guérir avec inscription de tel ou tel verset? Cela donne donc des indications sur ces pratiques, qu'il faut évidemment pouvoir recontextualiser par ailleurs, mais qui témoignent du fait qu'elles ont suscité une interrogation nécessitant une réponse juridique pour savoir ce qui était autorisé ou non et dans quel cadre. Concernant l'utilisation d'amulettes et de talismans ou les pratiques de guérison, comme l'usage d'incantations prophylactiques pour soulager tel ou tel problème, les questions et les réponses données sont généralement similaires à celles du médecin. On voit qu'il y avait une réelle demande d'incantations prophylactiques, d'amulettes ou de talismans qui complétaient ou se substituaient à la médecine. Le lien avec la médecine est bien évidemment très important dans ces pratiques.

A.-L. G.-C.: Les talismans trouvés dans la décharge à Dakar, de même que ceux décrits dans le Shams al-ma 'ārif, ont en partage une fonction de pharmakôn. Que sait-on du rapport exact que ces écrits talismaniques entretiennent avec le corps? Certaines amulettes, comme celle de la laveuse que vous avez citée, sont portées dans les cheveux, d'autres autour du cou ou suspendues à la ceinture. Leur usage semble s'accompagner du respect d'une hygiène corporelle variant en fonction des indications. Quelle relation ces écritures tissent-elles avec le corps des usagers?

A. E.: Les écritures peuvent être dites et soufflées sur un corps. L'écriture peut être transformée en liquide par rinçage de la tablette, immersion du papier ou autre dans de l'eau, dont il s'agira d'oindre intégralement le corps sans oublier un seul fraǵment de peau, ou de l'absorber par la bouche. Des indications portent sur le devenir du manuscrit plié qui a été donné par le marabout-scribe, où il est dit de le mettre dans un tissu blanc, rouge, ou multicolore, de le gainer d'un cuir de chèvre tanné ou au contraire à poils externes bruts de telle ou telle couleur, d'y associer des chiffres, d'y associer d'autres matières. Le lieu de suspension de l'amulette et de la mise en branle de son efficacité est prescrit par le marabout: Tu l'accroches à la ceinture, à la tête, aux reins, à la jambe, en une place précise d'un véhicule ou d'une maison, près d'une partie du corps ou de l'espace qu'il s'agit de renforcer, de blinder, de protéger. Beaucoup de ces objets ont pour but d'obtenir un blindage qui rende votre corps imperméable aux mauvaises paroles, au mauvais œil, aux coups de couteau, aux objets maléfiques lancés à distance.

\section{A.-L. G.-C.: Ces talismans peuvent être des boucliers contre de mauvaises paroles, dites-vous. Le caractère performatif de l'écriture est donc lié à la manipulation corporelle de l'objet-texte par les usagers.}

A. E.: Le même type d'amulette va servir à protéger les corps proprement dits, l'espace domestique, mais également le moyen de transport, l'automobile, la charrette, le cheval, l'âne, c'est-à-dire le corps en mouvement et l'unité avec laquelle le corps va se déplacer. Une amulette aura des usages différents selon l'inventivité et la foi de son propriétaire, mais aussi selon les niveaux de savoir, une même amulette peut être portée de façon passive, pour se protéger, mais aussi activée par une manipulation - par exemple l'inversion du portage de la gauche vers la droite -, ou une récitation complémentaire qui va la transformer en arme extrêmement puissante, renvoyant à l'expéditeur les maléfices ou les charmes qui vous étaient destinés. C'est très proche de ce qu'on peut voir chez des héros de bandes dessinées, porteurs d'armures défensives qu'un bouton ou une parole permet de transformer automatiquement en arme offensive surpuissante. Cette protection du corps fonctionne sur une parole, sur des ceintures talismaniques, dont le nombre de boucles peut aller jusqu'à quatre-vingt-dix-neuf. Mais c'est extrêmement rare, alors que l'on trouve très fréquemment des ceintures à une, deux, trois, sept boucles et plus. Il est dit que c'est une fois que l'ensemble de ces boucles sont fermées que l'amulette devient véritablement efficiente ou, inversement, qu'une ceinture amulette véritablement puissante est capable de se boucler elle-même en cas de danǵer. Une fois qu'on a mis en place ses amulettes, bouclé une ceinture, récité le nombre de fois ad hoc une formule coranique ou païenne appropriée, on est dans une espèce de cocon sensoriel, une espèce d'armure qui vous met complètement à l'abri, et qui vous permet éventuellement d'échapper au regard de vos adversaires.

\section{A.-L. G.-C.: Un talisman d'invisibilité, en somme. Le pouvoir attribué à ces amulettes est remarquable. Certaines amulettes auraient le pouvoir de déclencher une véritable fascination.}

\section{A. E.: Je pense aux manuscrits} d'amour et d'attirance, en particulier à la sourate de Yūsuf, ce fils d'un vieux monsieur, vraisemblablement polygame, martyrisé par ses frères qui l'ont soit abandonné soit vendu, et qui fait une très belle "carrière », puisqu'il finit ministre de pharaon et sauve toute sa famille, y compris ses frères traîtres... La première fois que je l'ai rencontrée, c'était auprès de l'un de mes premiers amis-informateurs-assistants-maîtres qui m'expliquait qu'il n'avait jamais pu lire cette sourate jusqu'au bout, tant elle était émouvante. Quelque part, c'était le mythe du migrant qui part loin de chez lui parce qu'il n'est pas forcément très aimé ou qu'il est abandonné par une partie de sa propre famille, et qui connaît une réussite absolument exceptionnelle. Ensuite, grâce à mes compères islamologues, Constant Hamès et Jean-Charles, nous avons retrouvé cette 
sourate de Yūsuf et identifié son efficacité en magie talismanique, dérivée de l'expression du désir amoureux de la femme du ministre vis-à-vis de Joseph [voir paraǵraphe suivant]. Il s'agit de simples mots, répétés, multipliés un certain nombre de fois, avec des occurrences mathématiques, des jeux de calliǵraphie. On les retrouve dans de nombreuses amulettes, que ce soit pour obtenir une emprise amoureuse sur quelqu'un indépendamment de son désir, ou s'aǵissant d'un politicien, d'un commerçant, pour attirer un maximum de personnes.

J.-C. C.: Ce qui est très intéressant avec cette sourate, c'est qu'elle aborde des sujets qui, même pour certains exégètes médiévaux, sont relativement frivoles. Il y aurait même un groupe khāridjite très minoritaire des débuts de l'islam qui, d'après des traités hérésiographiques, ne la considérait pas comme faisant partie du Coran, parce qu'elle abordait des sujets beaucoup trop frivoles pour pouvoir correspondre à la parole divine ${ }^{11}$. La figure de Joseph est très intéressante dans les traités de magie, c'est un prophète régulièrement mentionné. Je pense qu'il y a plusieurs raisons à cela. Il y a la thématique de cette sourate ${ }^{\mathbf{1 2}}$, mais aussi la figure de Joseph lui-même liée aux pratiques de l'oniromancie. Par exemple dans la sourate de Joseph, il y a un verset dans lequel il dit à son père:

"“Cher père, j'ai vu en songe onze étoiles, ainsi que le soleil et la lune devant moi se prosternant." Son père lui répondit: "Cher fils! ne conte point tes rêves à tes frères, sans quoi ils trameront contre toi une ruse, le Démon est pour l'homme un ennemi déclaré. » (Coran, 12, 4-5; traduction de Régis Blachère) On voit donc ici ses compétences en oniromancie ou onirocritique. Dans cette même sourate, il est question de la tentation de Joseph et de l'épouse de Putiphar, l'officier de Pharaon qui est attirée par lui. Deux passages principaux de cet épisode sont utilisés dans les traités de magie. Tout d'abord, la question de la tunique de Joseph, car, lorsque celui-ci est accusé d'avoir cherché à séduire l'épouse de Putiphar, il répond, je cite le verset coranique: "Mais Joseph interrompit: "C'est elle qui m'a tenté de ses charmes!" Un témoin de la famille de la dame attesta que si la tunique de Joseph était déchirée par-devant, la femme était sincère, tandis que Joseph était un menteur. Mais que si sa tunique était déchirée par-derrière, c'était elle qui mentait alors que Joseph était sincère. » (Coran, 12, 26-27 ; traduction de Régis Blachère) Ce motif de la tunique de Joseph se trouve dans certains traités de maǵie, presque comme un artefact au même titre que le sceau de Salomon, c'est-à-dire l'anneau qu'il portait et qui lui donnait pouvoir sur la création, ou le bâton de Moïse, grâce auquel il aurait séparé la mer en deux. Le Coran continue: "Ayant vu que la tunique était déchirée par-derrière, le maître de Joseph s'écria: "C'est là un fait de votre artifice de femmes! Votre artifice est immense!" (Coran, 12, 28)

L'autre épisode, très important dans les traités de magiie, et qui, je pense, a conditionné l'utilisation de la figure et de la sourate de Joseph dans les charmes d'amour, c'est lorsque les femmes d'Éǵgpte commencent à parler de cette affaire de la séduction de l'épouse de Putiphar. Le Coran dit: «Dans la ville cependant, des femmes dirent: "La femme du Puissant a tenté de ses charmes son valet, qui l'a percée d'amour pour lui, en vérité nous la voyons certes dans un égarement évident." Ayant ouï leur artifice, la coupable dépêcha quelqu'un vers elle, leur fit préparer des oranges et donna à chacune d'elles un couteau. “Entre auprès d'elles!" ordonna-t-elle à Joseph. Quand les femmes l'eurent aperçu, elles le trouvèrent si beau qu'elles se tailladèrent les mains dans leur émoi, et s'écrièrent: "À Dieu ne plaise! Ce n'est pas un mortel! C'est un noble archange! ”» (Coran, 12, 30-31; traduction de Régis Blachère) Cette scène où ces femmes sont subjuǵuées par la beauté de Joseph et se découpent les doigts associe à ce prophète un pouvoir séducteur presque surnaturel, que les fabricants de talismans essaient donc de s'approprier en utilisant les versets de cette sourate, et de cet épisode-là en particulier, dans la composition d'incantations ou de talismans écrits.

A.-L. G.-C.: Les frontières entre les savoirs issus des traités de magie et ceux qui relèvent de l'exégèse des textes coraniques semblent ténues dans l'islam médiéval. La figure de Joseph est présente à la fois dans la douzième sourate du Coran et dans les traités de sciences occultes. Elle s'offre comme un support d'interprétations nouvelles et de reformulations par les auteurs des écrits talismaniques. Cette créativité affleure dans la composition de ces écrits qui combinent non seulement les citations de versets extraits de sourates, mais aussi des noms, des chiffres ou des lettres. Par exemple dans le cas du «talisman des sept pactes de Salomon», nous avons affaire à une liste de noms se référant à des entités invoquées dans un ordre précis: djinns, anges, démons. Que sait-on de cette composition?

J.-C. C.: Dans le cas présent, dans cette liste de noms à la fin du talisman, il ne s'agit pas de noms de démons ou de djinns, mais plutôt de noms d'anges, de noms divins, et de ce qu'on appelle des noms «barbares», c'est-à-dire des mots qui n'ont pas de signification en arabe, sans être non plus des noms de démons. La plupart sont toutefois bien des noms d'anges : cela se reconnaît à la terminaison «-'ill»-dite suffixe théophore -, correspondant à des noms d'anges et que l'on retrouve dans nos prénoms qui en sont dérivés, que ce soit Gabriel (Djibrīl en arabe), Raphael (Isrāfīl en arabe) ou Michel (Mīkāīll). Parmi les autres noms, nous trouvons des noms divins et d'autres formés sur le modèle des premiers par assonance et allitération. Il ne s'agit donc pas, dans ce cas particulier, de l'invocation de démons ou de djinns.

Cependant, dans d'autres versions de ce talisman, figure l'invocation de rois des djinns. Les démons sont en revanche très peu sollicités puisque c'est une puissance dangereuse, alors que le djinn n'est ni bon ni mauvais, il peut être croyant ou mécréant. Et ce sont évidemment plutôt les rois des djinns, done des djinns qui ont pouvoir sur d'autres djinns et qui sont soumis à l'autorité d'un ange. Ainsi, il ne s'agiit pas forcément de djinns malfaisants, ni nécessairement plus bénéfiques ou bienveillants que les anges. L' «effrit» est aussi un cas particulier de djinn, d'une catégorie réputée spécialement puissante et souvent associée à Salomon, notamment parce que la seule occurrence d'un effrit dans le Coran est en lien avec celui-ci. Le terme de démon est toutefois très peu utilisé pour désigner les entités dont on sollicite une action.

A.-L. G.-C.: Le nom d'une chose ou d'un être est considéré, d'après l'œuvre d'Ibn 'Arabī dédiée à la science des lettres, comme l'expression de son essence. Pierre Lory, éminent spécialiste de mystique musulmane qui s'est penché sur cette question, affirme que «l'ensemble des lois régissant I'univers obéirait à une sorte de syntaxe universelle, comprenant ses noms (les essences), ses adjectifs (les accidents), ses verbes (les processus de 


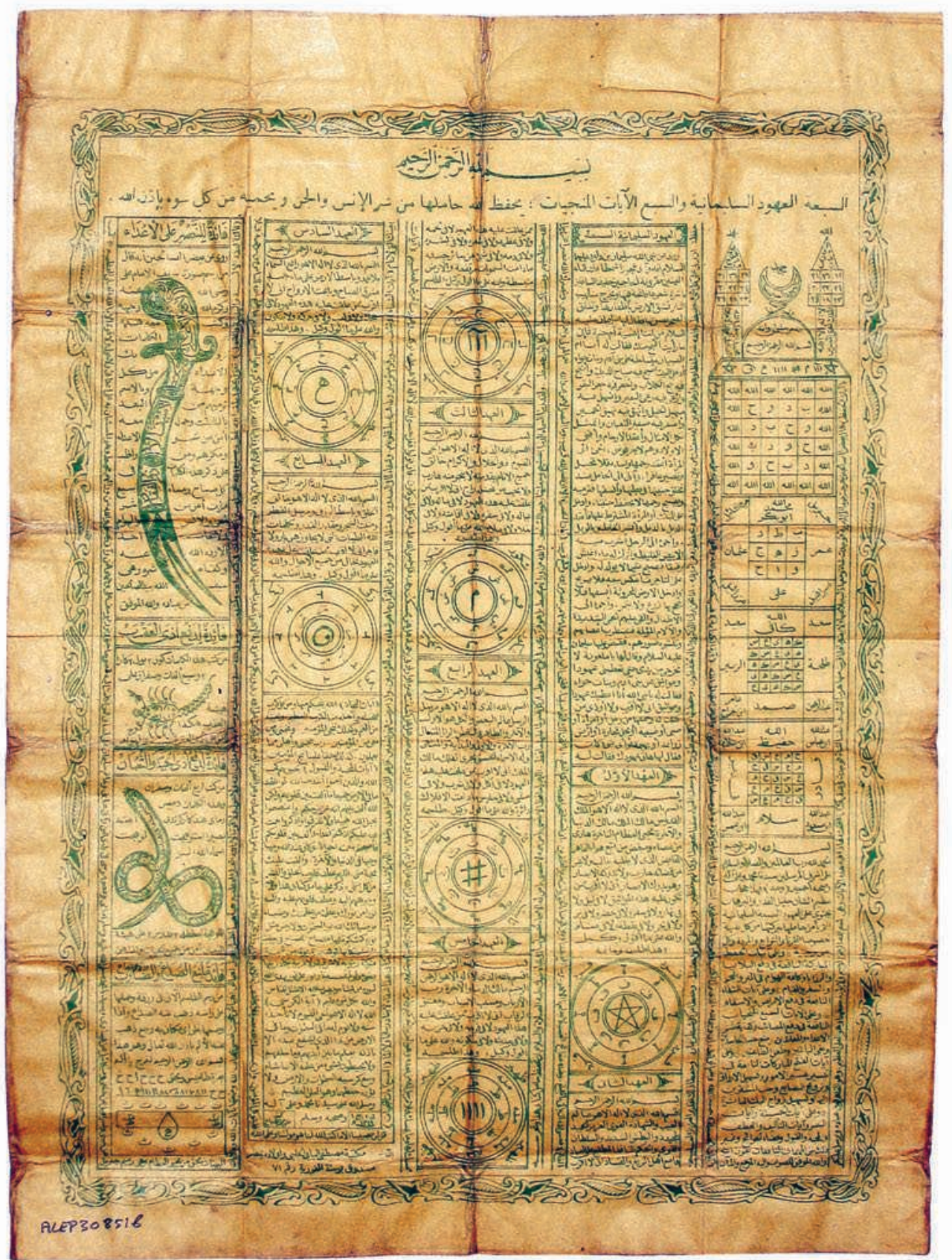

Talisman imprimé, initialement lithographié, extrait d'une amulette carrée gainée de cuir. Il s'agit du «talisman des sept pactes de Salomonछ, racontant comment Salomon contracta sept pactes avec la terrible Umm al-Șiby ( $n$ grâce auxquels elle ne pourrait pas s'en prendre aux humains. Collection ALEPEamulettes et objets magiques collectés dans les ordures (Mbebess Joal, Mbour, Sénégal, Afrique, Europe) par Alain Epelboin, 1983-2014. ALEP30851b. 

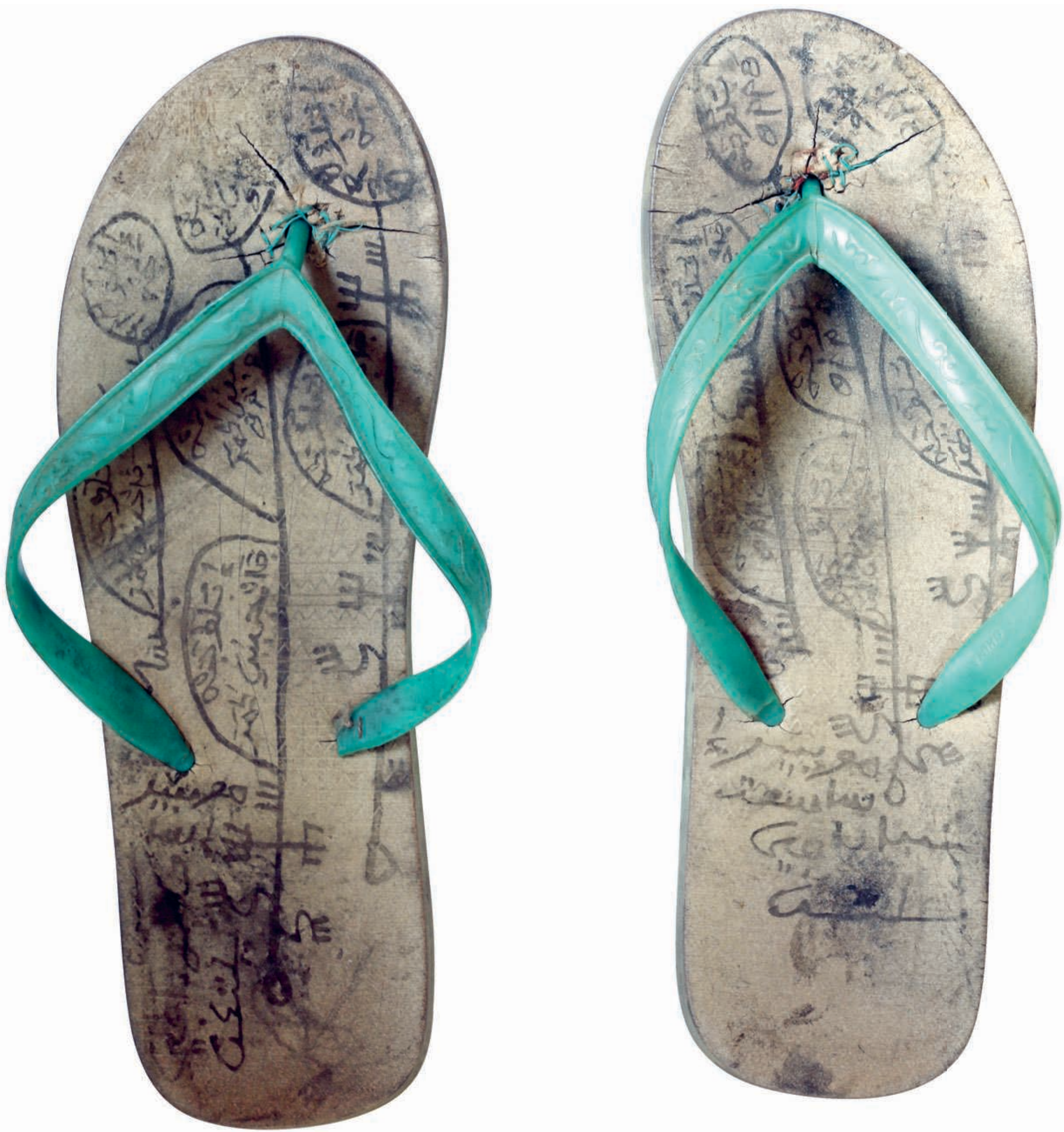

Sandales avec Dcritures talismaniques coraniques.

Collection ALEP: amulettes et objets magiques collectDs dans les ordures (Mbebess Joal, Mbour, SDnDgal, Afrique, Europe) par Alain Epelboin, $1983-2014$. ALEP00527. 
transformation)» (2012: $\left.165^{13}\right)$. Chaque lettre est investie d'un pouvoir particulier provenant de sa liaison avec la structure de l'univers. D'après cette conception analogique, le verbe magique serait un reflet de cette composition, de cette sagesse divine qui structure l'univers. La signification attribuée à ces listes de noms ou de lettres que l'on retrouve sur les écrits talismaniques n'apparaît ainsi clairement à l'exégète qu'une fois située dans ces réseaux de correspondances entre le langage et la création. Reste une énigme: qu'est-ce qui distingue alors ces invocations de noms d'anges, véhiculées par les écritures talismaniques, de cet autre registre familier que constitue la prière?

J.-C. C.: Il y a un rapport très ambigu entre la prière et les talismans ${ }^{14}$. En islam, il y a ǵlobalement deux types de prière : celle qu'on appelle șalāt, qu'on pourrait traduire par "prière canonique», qui est encadrée dans la jurisprudence islamique. Il existe des traités entiers consacrés à la manière de faire telle ou telle prière. Les cinq prières journalières sont des șalāts. Il y a aussi des cas particuliers de șalāt. Par exemple, celle qu'on va faire si on part en voyage. Il existe même une șalāt pour faire tomber la pluie, qu'on appelle șalāt al-istisqā. À côté de ces prières institutionnelles et canoniques encadrées par le droit, on en a d'autres tout à fait reconnues mais moins codifiées que sont les $d u(\bar{\alpha}$, qu'on peut traduire par «invocation». Or, ce terme sert également à désignner des invocations prononcées en vue d'obtenir un effet particulier et le texte de certains talismans est parfois lui-même appelé $d u(\bar{a}$. Par exemple, le texte du talisman de la laveuse est considéré comme un $d u(\bar{\alpha}$. On traduit par «invocation », "prière », le terme en arabe désignant une prière tout à fait acceptable, même si elle ne relève pas de la prière canoniquement codifiée. Ces $d u(a)$, qui sont des prières surérogatoires, autorisent une grande liberté de composition et peuvent intégrer des noms d'anges, des noms divins et des éléments un petit peu extérieurs. Pour donner un autre exemple sur ce rapport ambigu, le célèbre traité soufi intitulé Qūt al-qulūb (La Nourriture des cœurs) composé par Abū Țālib al-Makkī au $\mathrm{X}^{\mathrm{e}}$ siècle contient de nombreux $d u^{\prime} \bar{\alpha}$ - donc ces prières surérogatoires -, dont certains contiennent des éléments n'ayant pas de sens propre en arabe, mais qui sont très utilisés dans des prières magiques, comme des noms divins hébreux tels qu'Āl Shaddāy, Adūnāy, Ișbawt, etc. Ces noms-là ne se trouvent normalement pas dans des prières canoniques mais sont par contre tout à fait utilisables dans ce type de $d u(\bar{a}$.

\section{A.-L. G.-C.: Ce qui fait donc de ces talismans des écrits notoirement opaques aux non-initiés et autres usagers que le prescripteur ou le scribe. C'est intéressant. L'efficacité des invocations a-t-elle partie liée avec la possibilité d'avoir recours à un langage crypté qui mobilise des noms appartenant à d'autres traditions religieuses, en particulier aux traditions du monde hébraïque? On voit ainsi l'écrit magique tisser des liens en filigrane avec des traditions à la fois anciennes et extérieures au monde musulman.}

J.-G. C.: En fait, une des limites qui sont posées et qu'on trouve justement dans certains textes juridiques, notamment des fatwas, est que, pour ce genre de prière ou d'écrits, il ne faut normalement jamais utiliser des noms que l'on ne comprend pas, car le praticien ne peut pas savoir si c'est le nom d'un démon ou de quelque chose de malfaisant. Dans certains traités de sciences occultes, à la mention de ce genre de noms, l'auteur en précise systématiquement le sens ou l'utilisation spécifique. On a des sections entières sur des noms mystérieux, avec pour chacun une utilisation donnée. Parfois il n'est pas clair s'il s'agit pour l'auteur d'une explication du nom, d'une traduction ou d'un usage particulier. Ainsi, idéalement, le praticien est censé savoir ce qu'il écrit, mais est-ce toujours le cas? Les praticiens connaissaient-ils le sens de ces mots ou celui que leur donnaient les auteurs de traités magiques? Il est impossible de le dire, d'autant plus que, pour un certain nombre de ces noms, on ne trouve aucune explication dans les textes aujourd'hui connus. Découvrira-t-on un jour des textes qui en éclairent le sens ou bien cette connaissance est-elle perdue - si tant est qu'elle ait existé un jour? Il demeure en tout cas très difficile d'établir le niveau de connaissance du praticien quant aux éléments qu'il utilise dans son travail.

\section{A.-L. G.-C.: La connaissance de ces noms secrets et de leur traduction n'est accessible}

qu'à un cercle restreint d'initiés. Le caractère crypté de ces écrits les distingue du Coran, malgré I'inspiration que les auteurs de ces écrits talismaniques $y$ puisent. Comment l'usage de ces écrits talismaniques est-il alors accueilli en islam?

J.-C. C. : Il y a des avis divergents sur ces questions, parce qu'il n'y a pas d' «autorité centrale» en islam. Il serait présomptueux de vouloir établir une généralité pour $l^{\prime}$ «islam». Cependant, il faut se rendre compte qu'il y a des avis divergents, car il y a d'un côté la condamnation théorique de la magie, de l'autre une forte demande, et des populations qui utilisent et ont besoin de ce type de pratiques et d'objets. Je prendrai l'exemple d'une autre pratique, la ruqya, qu'on pourrait traduire par «incantation prophylactique», et qui peut parfois prendre la forme de $d u \bar{\alpha}$. Dans certains traités juridiques médiévaux - ce n'est pas systématique mais cela arrive souvent -, son statut est ambigu, c'est-à-dire qu'il n'y a pas d'indication formelle sur le caractère licite ou illicite, recommandé ou non, ce sont des indications de type «il n'y a aucun problème si c'est fait dans un but bienfaisant et que ça ne détourne pas de l'adoration de Dieu», ou alors «ce n'est pas autorisé sauf si c'est pour faire le bien et que ça ne détourne pas du culte de Dieu». Il existe donc une sorte de flottement, une permissivité en fonction de l'objectif et tant que l'on n'a pas recours à des puissances malfaisantes ou qui pourraient être considérées comme des concurentes à Dieu ou pouvant détourner le croyant de sa foi. Enfin, de nos jours, cela dépend également des pays ou des sensibilités reliǵieuses.

\section{A.-L. G.-C.: N'y a-t-il pas aussi une dimension subversive véhiculée par ces écrits talismaniques? Peut-on parler d'un usage privé, secret du texte qui se distinguerait d'un usage public et autorisé de ce dernier?}

J.-C. C. : Il est difficile de répondre à une telle question, car c'est une situation complexe et fort différente selon les époques et les régions. Pour la période mamelouke, c'est-à-dire du milieu du XIII ${ }^{\mathrm{e}}$ siècle au tout début du XvI ${ }^{\mathrm{e}}$, par exemple, le Shams al-máărif a été abondamment copié. Or, à cette époque, les sultans mamelouks financent des confréries soufies et font eux-mêmes appel à des cheikhs soufis dont certains sont versés en "science des lettres 
et des carrés maǵiques». On a des traces de sultans qui sollicitent justement les services de ces cheikhs pour leurs compétences «occultes». Cela fait partie de la pratique religieuse parce que lié à leur baraka propre et au savoir initiatique qu'ils ont reçu. Dans la mesure où les chroniques historiques en parlent, cela n'était $a$ priori pas complètement secret et connu des seuls arcanes du pouvoir. Mais, en revanche, on a aussi des textes qui condamnent ce type de savoir. L'un des exemples les plus célèbres est Ibn Khaldūn (m. 1406), qui considère tout ceci comme du soufisme dévoyé, corrompu, de la maǵie au sens négatif, presque de la sorcellerie qui ne s'assume pas comme telle et pour laquelle on utilise des mots soi-disant savants alors qu'il s'agirait, selon lui, de pratiques répréhensibles.

Les deux coexistent donc: à la fois des attestations de leur utilisation, des textes qui font la promotion de ce genre de savoir comme étant un savoir «initiatique» ou le summum du savoir et des connaissances, et des textes qui en condamnent l'usage. Il est ardu de distinguer ce qui pouvait réellement faire jurisprudence, dans tel ou tel contexte. Nous pouvons penser que ces sciences étaient tolérées tant qu'elles étaient au service du pouvoir ou se cantonnaient au milieu des élites, mais que le droit donnait un cadre plus contraignant, voire répressif, lorsqu'elles touchaient les populations, comme en témoignent des fatwas ou, pour certaines périodes, des décrets.

\section{$*$}

L'étude croisée des talismans arabes écrits du Sénégal d'aujourd'hui et des traités de sciences occultes arabes médiévaux mettent en évidence des permanences et des continuités: un certain nombre de textes, de sceaux, de noms, de versets coraniques témoignent d'une transmission des textes médiévaux jusqu'à nos jours. Les enquêtes respectives sur ces deux terrains sont source d'enrichissement mutuel: les pratiques d'aujourd'hui peuvent donner des pistes et hypothèses sur les silences des textes d'hier et les manuscrits anciens permettent de comprendre l'implicite des textes des talismans d'aujourd'hui dont les sources ne sont pas clairement identifiées, le praticien gardant ses connaissances sous le sceau du secret. Cependant, il faut bien entendu se préserver de toute projection des réalités d'une région et d'une époque sur l'autre: l'époque de production du corpus attribué à al-Būn̄i n'a absolument rien à voir avec celle des objets de la collection AlEp, leurs sociétés de production étant radicalement différentes. Ainsi, dans le premier cas, on a des textes composés dans des milieux lettrés et auprès des élites politiques, sans doute en contexte urbain, avec une diffusion restreinte en raison des modalités de copie et de fabrication des manuscrits. Dans le second, ces textes de talismans peuvent être écrits par des illettrés ou des personnes n'ayant pas la même formation que celle des élites mameloukes puis ottomanes; les «manuels», d'abord lithographiés puis imprimés, sont devenus bien plus facilement accessibles, permettant une réinterprétation ou une réappropriation de ces recettes et textes par un lectorat auquel il n'était pas destiné il y a quelques siècles. 
1. Alain Epelboin, Amulettes et objets magiques du musée de l'Homme, collectés dans les ordures du Sénégal: collection AIEP (Alain Epelboin), 1983-2016, catalogue interactif [en ligne], 2016, disponible surĐhttps://hal. archives-ouvertes.fr/hal-01285233 /document

Alain Epelboin etal., Collection AlEp: amulettes et objets magiques collectés dans les ordures (Mbebess Joal, Mbour, Sénégal, Afrique, Europe) 1983-2014 catalogue d'exposition [en ligne] ( «Dn art secretघles écritures talismaniques de I'Afrique de I'Ouest甘, Tourcoing, IMA en Nord-Pas-de-Calais, sept.-déc. [2014), 2014, disponible sur冋https://hal.archives-ouvertes. fr/hal-01081672/document

2. Alain Epelboin, Constant Hamès et Anne Raggi, «Cinq tuniques talismaniques récentes en provenance de Dakar (Sénégal)曰, in Constant Hamès (dir.), Coran ettalismans: textes et pratiques magiques en milieu musulman, Paris, Karthala, 2007घ147-174.

3. Alain Epelboin, «Bourquoi les amulettes sont-elles e) caces in Évelyne Heyer (dir.), Une belle histoire de l'Homme, Paris, Flammarion, 2015घ32-33.
4. Sur al-BDn\$̧et ses œuvres, voir日 La Magie en terre d'islam au Moyen Âge, Paris, Comité des travaux historiques et scientifiques, 2017, Noah Gardiner, «Eorbidden Knowledge? Notes on the Production Transmission, and Reception of the Major Works of Ahmad al-BDns Journal of Arabic and Islamic Studies12, 2012:81-143!Noah Gardiner, «Bsotericist Reading Communities and the Early Circulation of the Sufi Occultist Ahmad al-BDn\$s Works凹, Arabica 64 (3-4), 2017:405-441.

5. Le premier grand texte qu a fait connaître le Shams al-ma'ārif au public européen est l'ouvrage d'Edmond Doutté, Magie et religion dans I'Afrique du Nord, Alger, A. Dourdan, 1909.

6. Sur ce talisman et la figure de $\mathrm{H}$ ( rDn al-Rash\$̣d dans les sciences occultes islamiques, voir日 Jean-Charles Coulon, «"Da figure de $\mathrm{H}$ ( rDn al-Ra Có̀d dans la tradition magique islamique区, in Catherine Pinon (éd.), Savants, amants, poète etfous: séances offertes à Katia Zakharia, Beyrouth, Presses de l'Ifpo, 2019घ175-231.

7. Sur H( rDn al-Rash\$d dans les Mille et Une Nuits, voirĐocelyne Dakhlia, «Dne vacance califale日
Hârûn al-Rashîd dans Les Mille et Une Nuits区, in Aboubakr Chraïbi (dir.), Les Mille et Une Nuits en partage, actes du colloque (Paris, Fondation Singer-Polignac et Inalco mai 2004), Paris, Sindbad-Actes Sud, 2004E168-181.

8. Sur les références à la littératur arabe ou à I'histoire dans le Shams al-ma'árif, voirØlean-Charles Coulon, «\$Magie et politiqueĐévénements historiques et pensée politique dans le Šams al-ma'ärif attribué à al-BDn\$(mort en 622/1225) 区 Arabica 64 (3-4), 2017घ442-486.

9. Voir par exemple la tunique du grand vizir Qar( Mușțaf(

et la tunique de guerre ottomane présentées par Philippe

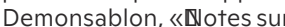
deux vêtements talismaniques区 Arabica日3 (2), 1986घ216-250.

10. Sur les bols talismaniques, voir par exempleUTawfiq Canaan «प̣̂asit er-radjfeh (fear cup) 曰 Journal of Palestine Oriental

StudiesB, 1923日122-131!Tawfiq Canaan, «Arabic Magic Bowl区, Journal of Palestine Oriental Studies[6, 1936घ79-127घHans Henry Spoer, «Brabic Magic Medicinal Bowls区, Journal of the American Oriental Society 65,1935 237-256, 258, 1938घ366-383घHans
Henry Spoer, «Arabic Magic Bowls I. An Astrological Bowl घ, Journal of the American Oriental Society日8, 1938घ366-383@Sigismund Sussia Reich, «Quatre coupes magiques曰 Bulletin d'études orientales వ-8, 1937-1938घ159-175日Annette Ittig, «B Talismanic Bowl , Annales islamologiques[18, 1982日79-94, Giovanni Canova, «"lațāsatal-ism: note su alcune coppe magich yemenite匹, Quaderni di Studi

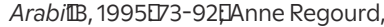
«Deux coupes magiques, biens de fondation pieuse (nord du Yémen) transmission du savoir ete) cacité甘, in Constant Hamès (dir.), Coran et talismans: textes et pratique magiques en milieu musulman, Paris, Karthala, 2007घ309-345.

11. G. Levi Della Vida

« (dir.), Encyclopédie de l'Islam,

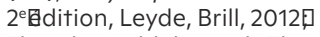
Theodor Nöldeke et al., The History of the Qur'ān, trad. de l'allemand par Wolfgang H. Behn, Leyde/ Boston, Brill, 2013日288घ al-Shahrast( $n \$$ Livre des religions et des sectes, t.L trad. de l'arabe par Daniel Gimaret et Guy Monnot, Louvain, Peeters-Unesco, $1986 \mathbb{B} 97$.

12. Sur la sourate de Joseph en général, voirĐAlfred-Louis De Prémare, Joseph et Muhammad, le chapitrexII du Coran, Aix-enProvence, Publications de I'université de Provence, 1989.

13. Pierre Lory, «Bensées magiques en islam曰, Cliniques méditerranéennes85, 2012日 163-174.

14. À ce sujet, voir notammentu Constant Hamès, «Entre recette magique d'al-Bûnî et prière islamique d'al-GhazaliEtextes talismaniques d'Afrique occidentaleß Systèmes de pensée en Afrique noire代, 1993 187-223. 